Abstract E-033 Table 1 Baseline characteristics of enrolled with acute M2 occlusion

\begin{tabular}{|c|c|c|c|c|c|}
\hline Characteristic & $\begin{array}{c}\text { M2 } \\
<1.63 \mathrm{~mm} \\
(\mathrm{~N}=26)\end{array}$ & $\begin{array}{c}\mathrm{M} 2 \\
1.62-1.95 \mathrm{~mm} \\
(\mathrm{~N}=26)\end{array}$ & $\begin{array}{c}\mathrm{M} 2 \\
1.95-2.29 \mathrm{~mm} \\
(\mathrm{~N}=26)\end{array}$ & $\begin{array}{c}\mathrm{M} 2 \\
>2.29 \mathrm{~mm} \\
(\mathrm{~N}=26)\end{array}$ & $P$ \\
\hline Age, mean \pm SD & $72.0 \pm 10.1$ & $71.1 \pm 10.7$ & $71.3 \pm 10.6$ & $66.4 \pm 15.3$ & 0.30 \\
\hline Male, $n(\%)$ & $13(50.0)$ & $15(57.7)$ & $13(50.0)$ & $15(57.7)$ & 0.89 \\
\hline $\begin{array}{l}\text { Procedure time (hr), } \\
\text { median (IQR) }\end{array}$ & $\begin{array}{c}0.80 \\
(0.30-1.35)\end{array}$ & $\begin{array}{c}0.80 \\
(0.50-1.13)\end{array}$ & $\begin{array}{c}0.50 \\
(0.38-0.80)\end{array}$ & $\begin{array}{c}0.70 \\
(0.48-1.15)\end{array}$ & 0.24 \\
\hline $\begin{array}{l}\text { Initial NIHSS, } \\
\text { median (IQR) }\end{array}$ & $8(5-15)$ & $10(5-16)$ & $11(8-19)$ & $14(8-19)$ & 0.11 \\
\hline \multicolumn{6}{|l|}{ Complication, n (\%) } \\
\hline Extravasation & $4(15.4)$ & $1(3.8)$ & $0(0)$ & $1(3.8)$ & 0.10 \\
\hline Vasospasm & $2(7.7)$ & $1(3.8)$ & $1(3.8)$ & $0(0)$ & 0.29 \\
\hline Dissection & $2(7.7)$ & $1(3.8)$ & $0(0)$ & $0(0)$ & 0.29 \\
\hline $\begin{array}{l}\text { Hemorrhagic } \\
\text { transformation }\end{array}$ & $11(42.3)$ & $7(26.9)$ & $5(19.2)$ & $3(11.5)$ & 0.07 \\
\hline $\mathrm{SAH}$ & $9(34.6)$ & $3(11.5)$ & $1(3.8)$ & $0(0)$ & 0.001 \\
\hline ICH & $1(3.8)$ & $2(7.7)$ & $4(15.4)$ & $1(3.8)$ & 0.36 \\
\hline IVH & $1(3.8)$ & $2(7.7)$ & $1(3.8)$ & $0(0)$ & 0.56 \\
\hline TOAST, n (\%) & & & & & 0.43 \\
\hline LAD & $7(26.9)$ & $4(15.4)$ & $5(19.2)$ & $7(26.9)$ & \\
\hline Cardioembolism & $16(61.5)$ & $14(53.8)$ & $13(50.0)$ & $13(50.0)$ & \\
\hline Other determined & $0(0)$ & $5(19.2)$ & $4(15.4)$ & $1(3.8)$ & \\
\hline Undetermined & $3(11.5)$ & $3(11.5)$ & $4(15.4)$ & $5(19.2)$ & \\
\hline $3 \mathrm{mo} . \mathrm{mRS}, \mathrm{n}(\%)$ & & & & & 0.36 \\
\hline $0-2$ & $14(53.8)$ & $12(46.2)$ & $11(42.3)$ & $17(65.4)$ & \\
\hline
\end{tabular}

using axial source image of CT or MR angiography conducted before EVT. The association of M2 diameter with clinical and procedural outcome was assessed. Procedural complication was captured including extravasation during angiography and hemorrhagic transformation on follow up image.

Results A total of 104 patients (age, $70.2 \pm 11.9$ years; male, 53.8\%) was evaluated. Arterial diameter of M2 segment was ranged from 1.05 to $3.19 \mathrm{~mm}$. Six patients were treated with intraarterial urokinase only, 90 with stent retriever, 3 with aspiration thrombectomy, and 5 patients with both stent retriever and aspiration thrombectomy. Extravasation developed four times more frequently in the lowest quartile group $(<1.63 \mathrm{~mm})$ than the highest quartile group $(\geq 2.29 \mathrm{~mm})$ divided by $\mathrm{M} 2$ diameter. Hemorrhagic transformation occurred in $25 \%$ of patients and the half of them was subarachnoid hemorrhage. In the logistic regression analysis, the lowest quartile group showed positive association with hemorrhagic transformation (adjusted ORs 8.91 [95\% CIs 1.50 - 52.93]), but no association with clinical outcome.

Conclusions This study demonstrated that the lowest quartile of M2 diameter was associated with procedural complication in the acute M2 occlusion patients treated with EVT. However, clinical outcome was not differed.

Disclosures J. Kim: None. K. Lee: None. J. Kang: None. B. Kim: None. M. Han: None. H. Bae: None. S. Baik: None. L. Sunwoo: None. J. Kim: None. C. Jung: None.

\section{E-034 \\ ENDOVASCULAR TREATMENT OF WIDE-NECK ANEURYSMS, AN EVALUATION OF SAFETY AND EFFECTIVENESS OF STRYKER SURPASS EVOLVETM FLOW DIVERTER SYSTEM}

${ }^{1} \mathrm{~A}$ Arthur* ${ }^{*}{ }^{2} \mathrm{~J}$ Fifi, ${ }^{3} \mathrm{H}$ Rice, ${ }^{4} \mathrm{~A}$ Wakhloo, ${ }^{5} \mathrm{~A}$ Coon, ${ }^{6} \mathrm{~V}$ Pereira. ${ }^{7}$ Neurosurgery, University of Tennessee Health Sciences Center and Semmes-Murphey Clinic, Memphis, $T N_{;}{ }^{2}$ Icahn School of Medicine at Mount Sinai, New York, NY; ${ }^{3}$ Department of Interventional Neuroradiology, Gold Coast University Hospital, Southport Queensland, Australia; ${ }^{4}$ Department of Neurointerventional Radiology, Beth Israel Lahey Health, Burlington, MA; ${ }^{5}$ Carondelet Neurological Institute, St. Joseph's Hospital, Tuscon, AZ; ${ }^{6}$ University Health Network, University of Toronto, Toronto, ON, Canada

\subsection{6/neurintsurg-2021-SNIS.130}

Introduction Stroke is the most common life-threatening neurological disease and the fourth leading cause of death in the United States. Each year in the U.S. 795,000 people suffer a stroke. Hemorrhage is responsible for $13 \%$ of these events and the majority are related to the rupture of intracranial aneurysms. The incidence of unruptured intracranial aneurysms (IA) in the general population has been estimated to range from approximately $2 \%$ up to $6 \%$. Data available on patients with subarachnoid hemorrhage indicated that the majority are from smaller aneurysms, although many smaller aneurysms are appropriately treated conservatively. Literature suggests that 80 to $90 \%$ of all ruptured intracranial aneurysms are less than or equal to $12 \mathrm{~mm}$ in size. Outcomes derived from Surpass Streamline treatment have been reported for large and giant aneurysm populations through the SCENT study, however 
outcomes for small and medium aneurysms for aneurysm locations including internal carotid artery (ICA) and distal to ICA terminus are unknown.

Methods The EVOLVE study is a prospective, historical-control based, single arm, multicenter clinical trial delineating the safety and efficacy of the second generation Surpass EVOLVE Flow Diverter System for the treatment of wide-neck $(\geq 4 \mathrm{~mm}$ or no discernible neck), small and medium sized saccular or fusiform IA $\leq 12 \mathrm{~mm}$ located on the ICA and its branches with a reference vessel diameters 2.5 to $5.0 \mathrm{~mm}$.

Approximately 235 patients will be enrolled at approximately 40 investigational sites located in the USA, Canada, and Australia. Clinical follow-up is planned at discharge, 30 and 180 days and at 1, 2, and 3 years. All subjects will undergo DSA imaging at 1 - and 3 -years post device implantation. The respective primary safety and efficacy endpoints are neurological death or disabling stroke at 12 months, as assessed by an independent CEC; and the composite of Raymond I occlusion with no significant (>50\%) parent artery stenosis, and no target aneurysm retreatment; as assessed by an independent Core Lab. An analysis of non-inferiority to pre-established safety and effectiveness performance goals will be completed following 12-month follow-up.

Other key outcomes include assessment of procedural technical success, major stroke, minor stroke, TIA, stent thrombosis, recanalization, durability of occlusion and occlusion status. Conclusion The EVOLVE prospective, multicenter, single arm, pre-market study (ClinicalTrials.gov NCT: NCT04195568), is designed to evaluate the safety, efficacy, and performance of the Surpass EVOLVE Flow Diverter System in the treatment of unruptured wide-neck, saccular or fusiform IA located in the ICA and its branches with an RVD of 2.5 to $5.0 \mathrm{~mm}$.

Disclosures A. Arthur: 2; C; Stryker Neurovascular. J. Fifi: 1; C; Stryker Neurovascular. 2; C; Stryker Neurovascular. H. Rice: 2; C; Stryker Neurovascular. A. Wakhloo: 2; C; Stryker Neurovascular. A. Coon: 2; C; Stryker Neurovascular. V. Pereira: 2; C; Stryker, Medtronic, Balt, Cerenovus.

\section{E-035 SAFETY AND EFFICACY OF STENT-ASSISTED COILING OF RUPTURED WIDE NECK ANEURYSMS IN COMPARISON TO BALLOON ASSISTED COILING IN ACUTE SUBARACHNOID HEMORRHAGE} ${ }^{3} \mathrm{~A}$ Mendez Ruiz, ${ }^{3} \mathrm{C}$ Zevallos, ${ }^{3} \mathrm{D}$ Quispe-Orozco, ${ }^{3} \mathrm{M}$ Farooqui, ${ }^{2} \mathrm{~J}$ Delgado Almandoz, ${ }^{3} \mathrm{~S}$ Ortega-Gutierrez*. ${ }^{1}$ Aurora Neuroscience Institute, Milwaukee, WI; ${ }^{2}$ Abbott Northwestern Hospital, Minneapolis, MN; ${ }^{3}$ University of lowa Hospitals and Clinics, lowa City, IA; ${ }^{4}$ University of Pittsburgh Medical Center, Pittsburgh, PA

10.1136/neurintsurg-2021-SNIS.131

Introduction Ruptured wide neck aneurysms (RWA) have proven challenging for acute endovascular embolization and are still preferentially treated with microsurgical clipping in most institutions. Balloon assisted coiling (BAC) has been used for acute embolization of RWA with some success in recent past but with high recanalization rates requiring re-treatment, risk of parent vessel coil protrusion, and thromboembolic complications. The aim of this study is to assess the safety and efficacy of using Stent assisted coiling (SAC) and/or Balloon assisted coiling (BAC) for acute embolization of RWA.

Methods We performed a retrospective analysis of data at two comprehensive stroke centers (CSC) for patients with RWA who underwent endovascular treatment with SAC and BAC between 2010 and 2020. Patient selection for either treatment category was at the discretion of the treating physician. The treating physician also made the decision in case of SAC, whether to load the patient with dual oral antiplatelets or place them on intravenous antiplatelet regimen. Primary clinical outcomes of interest were modified Rankin Scale (mRS) at 3-months and Raymond Roy (RR) Occlusion Classification, immediately post-operative and at 3 -months. Patient demographics, clinical presentation (Hunt and Hess score $\{\mathrm{HH}$, modified Fisher scale $\{\mathrm{mFS}\}$ and Glasgow coma scale $\{\mathrm{GCS}\}$ ), comorbidities, aneurysms characteristics, technical success, complications, and clinical and imaging follow-ups were also assessed. Univariate and multivariable logistic regression and ordinal regression was used to compare the primary and secondary outcomes. All the statistical analysis was performed using R-software at an $\alpha \leq 0.05$ significance.

Results 112 patients were treated with SAC and 109 underwent BAC. There was no significant difference in baseline characteristics between the 2 groups with median age of 53.5 years in SAC and 55.81 years in BAC $(p=0.419)$, nor in their clinical characteristics with regards to $\mathrm{HH}, \mathrm{mFS}$, and GCS. There was no significant difference between the two groups with regards to aneurysm location $(77.7 \%$ vs $84.4 \%$ in anterior circulation, $\mathrm{p}=0.78$ ) or size. Coil herniation was significantly higher in BAC group $(9.2 \%$ vs $1.8 \%, \mathrm{p}=0.033)$ with no significant difference in other perioperative and in-hospital complications between the two groups including intracranial hemorrhages, strokes, groin hematoma requiring transfusions, and EVD related complications. There was no significant difference in mRS 3-6 at 3 months (38 vs 22, p=0.096). The SAC group had significantly higher number of patients with RR Class I, immediately post-operative (45 vs 26, $\mathrm{p}<0.001 \%$ ), with significantly less patients in RR Class II and III at 3 months follow up (29 vs $39, \mathrm{p}<0.001 \%)$. Similarly, after adjusting for the variables, there was no statistical difference in the mRS shift analysis $(p=0.062)$ and $R R(p=0.35)$ at 90 days among the two groups.

Conclusion In this study, we observed that SAC is as safe as BAC for acute treatment of RWA, with better aneurysm occlusion rates, less re-treatment and without an increase in hemorrhagic complications. Further studies are warranted to validate these findings in a prospective randomized manner before widespread application in the management of patients with acute aneurysmal subarachnoid hemorrhage.

Disclosures S. Dandapat: None. A. Wallace: None. G. Lopez: None. Y. Kayan: None. A. Mendez Ruiz: None. A. Copelan: None. A. Dajles: None. A. Mendez Ruiz: None. C. Zevallos: None. D. Quispe-Orozco: None. M. Farooqui: None. J. Delgado Almandoz: None. S. Ortega-Gutierrez: None.

\section{E-036 NEUROENDOVASCULAR INTERVENTIONS WITH TRANSCIRCULATION APPROACH: A CASE-SERIES APPROACH TO SOLVE CHALLENGING SITUATIONS}

${ }^{1} \mathrm{M}$ Colasurdo*, ${ }^{2} \mathrm{G}$ Edhayan, ${ }^{3} \mathrm{P}$ Kan, ${ }^{1} \mathrm{~K}$ Raghuram. ${ }^{1}$ Neuroradiology, University of Texas Medical Branch, Galveston, TX; ${ }^{2}$ Radiology, University of Texas Medical Branch, Galveston, TX; ${ }^{3}$ Neurosurgery, University of Texas Medical Branch, Galveston, TX

10.1136/neurintsurg-2021-SNIS. 132

Introduction With the development of advanced endovascular techniques and materials, neurointerventionalists are able to perform challenging and complex cases that were previously difficult to perform. Transcirculation approaches could be a 